\title{
CONSTRUCTION AND PERFORMANCE CHARACTERISTICS OF A RAPID DISCHARGE TEA $\mathrm{CO}_{2}$ LASER
}

\author{
G.J. ERNST and A.G. BOER \\ Twente University of Technology, Department of Applied Physics, \\ Enschede, The Netherlands
}

Received 19 June 1978

\begin{abstract}
A novel excitation technique is described for molecular gas lasers. A specially constructed electrode configuration is activated by a rapid Marx generator. A very homogeneous selfsustained discharge can be obtained although no separate preionization source is present. The construction is applied to a $\mathrm{CO}_{2}$ system. Details of the operating characteristics are given. We found the maximum energy production to be over $60 \mathrm{~J}$ per liter and a small-signal gain of $6 \%$ per cm could be reached.
\end{abstract}

\section{Introduction}

Since the discovery of the transverse excitation of the atmospheric-pressure $\mathrm{CO}_{2}$ laser by Dumanchin [1] and Beaulieu [2] several transverse excitation techniques have been developed, e.g. the Dumanchin, the Lamberton-Pearson and the ultraviolet and electron beam sustained types. The first three types have in common that UV radiation conditions the laser gas before and/or during the discharge. Sometimes doping with an organic compound of low ionization potential is added in order to help conditioning the gas. In all three types the UV radiation is produced in a separate discharge such as by means of trigger wires or by an external UV source. A drawback of all types of self-sustained discharges compared with the EB and UV sustained ones is the limited excitation rate resulting in a limited total energy dissipation. This has to do with the fact that one has to wait with applying the main voltage until the preionization is sufficiently homogeneous in order to avoid sparking between the electrodes. This is one of the reasons why self-sustained discharges are inferior to UV and EB sustained ones.

In this paper we describe a self-sustained singledischarge TEA system that differs from existing ones by the fact that no additional UV source is present and the excitation rate is very fast. We used an electrode gap of $20 \mathrm{~mm}$ and obtained with this system values for the power output and small-signal gain superior to other self-sustained discharges and in some respects even to UV and EB sustained ones. The system is scalable in principle, but to what sizes has not yet been investigated.

The system is an improved version of the Blumlein type construction, which is published as part of a thesis $[3,4]$. Particularly the reliability for $\mathrm{CO}_{2}$-rich mixtures is improved and this system is, in contrast to the former one, in principle scalable to larger electrode gaps. It is essentially a specially constructed electrode configuration activated by a very rapid power source. As a power source we designed a two-stage Marx generator with variable capacitance, having a rise time of about $15 \mathrm{~ns}$ (somewhat dependent on the applied voltage and capacitance). The operation of the system is very reliable under a large range of different gas mixtures without the need of an organic dopant. The discharge is very homogeneous without a single streamer, which results in a very high power output and a very large gain.

\section{Description and working principle of the system}

Fig. 1 gives the construction details of the laser system. We used highly polished aluminium profiles with a gap of $20 \mathrm{~mm}$. The electrodes are profiled ac- 


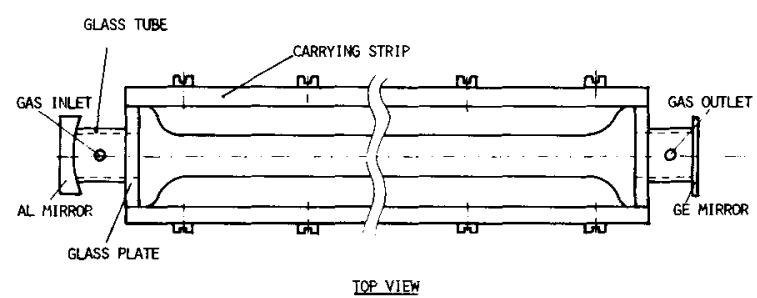

Fig. 1. Top view of the electrode configuration.

cording to a publication of Chang [5]. For his parameters $k$ and $v$ we chose $k=0.02$ and $v=\arccos$ $(-k)$ in order to get a square discharge. The height of the profiles is $10.7 \mathrm{~mm}$ and the corresponding width is $64 \mathrm{~mm}$, whereas the discharge length is $40 \mathrm{~cm}$. The profiles form an integral part with the carrying strips having a length of $50 \mathrm{~cm}$; these strips are held apart at the ends by two glass plates having a hole of $30 \mathrm{~mm}$ diameter in order to ensure rigidity and the proper distance between the electrodes. Each sidewall of the laser is formed by a heat-resisting glass plate of $2 \mathrm{~mm}$ thickness. At the front and rear side a glass tube is glued for carrying the high-reflecting aluminium mirror and the uncoated flat germanium outcoupling mirror. Also the gas inlet and outlet is through these tubes. The aluminium mirror has a $2 \mathrm{~m}$ radius of curvature. One of the electrodes is extended by two copper plates, which are bent alongside the glass plates, as is shown in fig. 2. Between those copper plates and the glass plates is some insulation material for preventing arcing between the copper plates and the other electrode. The inductance between the electrodes and the twostage Marx generator is kept as low as possible. When the Marx generator is fired a rapid voltage rise appears between the electrodes. Because of the short distance between the copper plates and the opposite polarity electrode a very high electric field strength with rapid rise appears between them. This results in a corona discharge of short duration $(10-30$ nsec, de-

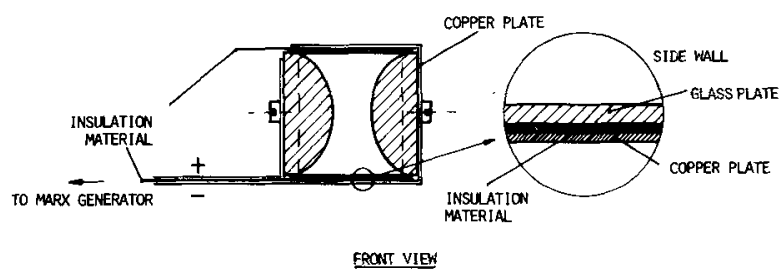

Fig. 2. Details of the side-wall construction of the laser system. pendent on the discharge conditions), which flows alongside the glass plates. The UV light emitted by thos discharge illuminates the electrode gap and conditions the main discharge, so that a uniform discharge is obtained between the electrodes. Because of the high field strength and the rapid rise of the voltage the UV source, which extends over the full size of the glass plates, is very strong. This results in an excellent glow discharge, in which no streamer can be found. This in turn improves the optical quality of the generated beam.

The most importance feature of the system is this built in UV source having a large area and very high homogeneity (in fact no corona discharge can be seen, which indicates that the corona is not of the streamer type). The proper operation of this UV source depends upon the distance between the electrodes and the copper plates and the width of the electrodes. When the field strength between the copper plate and the opposite polarity electrode is too low, the laser sparks between the electrodes because of insufficient preionization. For very high field strengths the corona discharge degrades into an arc along the surface of the glass plates between the electrodes. Although we did not investigate the tolerances under all circumstances, the thickness of the glass plates must certainly be between 1 and $4 \mathrm{~mm}$ and the electrode width between 63 and $66 \mathrm{~mm}$.

In order to prove that our explanation for the working principle by means of a corona discharge along the glass plates is correct we carried out some current measurements. A typical example of the behaviour of the current versus time is shown in fig. 3 . The vertical scale is about $550 \mathrm{~A} /$ div and the horizontal one is 50 $\mathrm{ns} / \mathrm{div}$. These measurements have been performed with a different electrode system giving a narrower and shorter discharge. The gas mixture we used was $\mathrm{CO}_{2}$ : $\mathrm{N}_{2}: \mathrm{He}=1: 1: 10$. We used such a He-rich mixture in order to perform the measurements at a relatively low voltage level, where little noise is present. A simple capacitor with $\mathrm{C}=0.02 \mu \mathrm{F}$ is used as a power source. It is connected to the electrodes through a spark gap having low self-inductance. The voltage applied was $26 \mathrm{kV}$. The current was measured across a $\mathrm{CuSO}_{4}$ resistor with a dynamical resistance of $0.9 \Omega$ placed in series with the laser. We observed experimentally that the relative height of both peaks can be changed for instance by varying the current rise time. For very short rise times the first peak will dominate. Under 


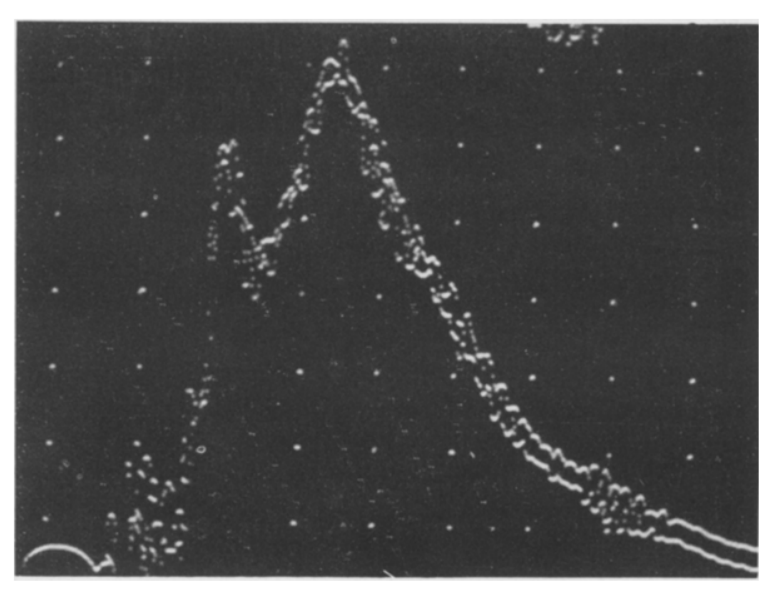

Fig. 3. The current as a function of time. The gas mixture is $\mathrm{CO}_{2}: \mathrm{N}_{2}: \mathrm{He}=1: 1: 10$ and the laser voltage $26 \mathrm{kV}$. At the vertical axis the current is plotted in $550 \mathrm{~A} / \mathrm{div}$. The horizontal axis is $50 \mathrm{~ns} /$ div. For further explanation: see text.

those circumstances (especially when the width of the electrodes is small) a corona discharge can be seen which flows along the glass plates. So the first peak has to be attributed to the corona discharge and the second one to the main discharge. Note the high current density of $85 \mathrm{~A} / \mathrm{cm}^{2}$ over our $1.5 \times 30 \mathrm{~cm}^{2}$ electrode surface. For higher voltages this current density is even much higher. From the observations it can be seen that the strength of the UV source is also very dependent on the rise time of the voltage. We observed sparking between the profiles for long rise times and arcing along the surface of the glass plates for short rise times. Proper working of the system will be obtained if the rise time lies between 10 and 50 ns. Why the corona discharge does not degrade into a spark in which the total energy of the system is dissipated is not fully clear. Obviously the ignition of the corona discharge along a dielectric is easily brought about because recombination between charged particles can take place so easily and also because of the high field strength between the right electrode and the copper plate. But once the discharge has been started, the dissipation is so high only a small amount of energy can be dissipated and the main discharge can take over.

In fig. 2 it can be seen that the right electrode is extended over both glass plates. This is not always necessary. Most of the time the upper copper plate can be omitted. However we found that in that case for He- rich mixtures the laser discharge is situated lower than the central position between the electrodes. This has also been observed in Blumlein-type $\mathrm{N}_{2}$ lasers [6] and there it was attributed to the dynamical effect of trying to minimize the self-inductance in the laser current loop and to stray ground capacitors. According to our investigations we must attribute this effect to the electric field distribution in the laser. The problem can easily be circumvented by symmetrizing the static electric field distribution.

\section{The output and gain measurements}

In order to be able to study the power output as a function of the applied voltage and the input energy we constructed a rapid Marx generator having variable capacitance. Induction loops and stray capacitances inside the Marx generator are kept as small as possible; specially constructed spark gaps are used having low self-inductance and the capacitors are ceramic type from Sprague Company. These capacitors are suitable for voltages up till $30 \mathrm{kV}$, but we used them up till

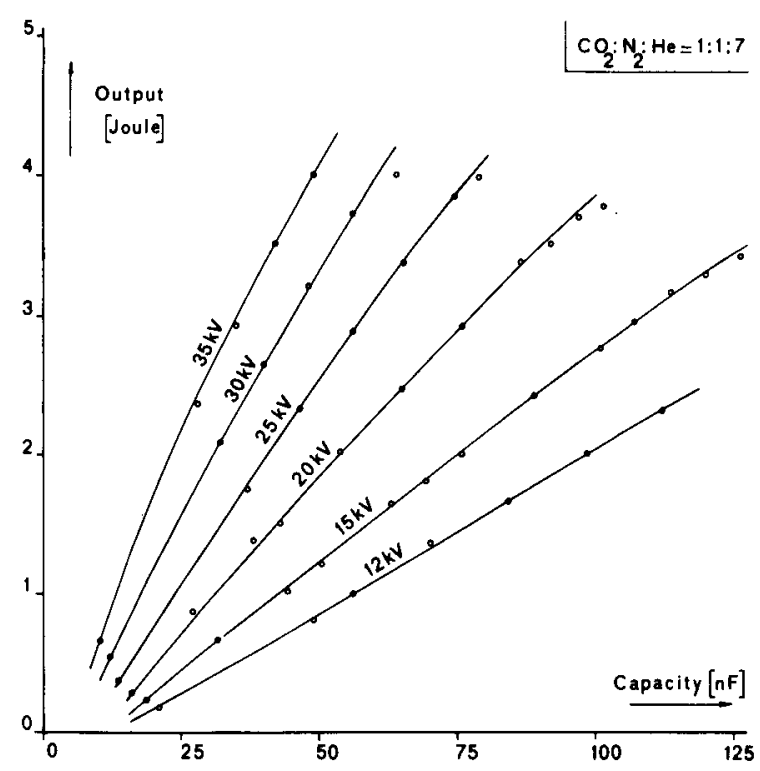

Fig. 4. The power output as a function of capacitance. At the vertical axis the output is plotted in $\mathrm{J} /$ div. At the horizontal axis the capacitance of one stage of the Marx generator is plotted. The parameter used in the figure is the voltage of one stage. The gas mixture is one part $\mathrm{CO}_{2}$, one part $\mathrm{N}_{2}$ and seven parts He. 
$40 \mathrm{kV}$. Because their capacity is very dependent on the applied voltage we measured it by determination of the stored energy as a function of the voltage applied. The capacity values at the horizontal axis of the output figures are the real capacitances. For measuring the power output we used a calorimetric laser power meter model nr. 38-0402 from Scientech Inc. gauged with a Gen-Tec type pyroelectric Joule meter model ED-500.

In figs. 4, 5, 6 and 7 the output characteristics are given as a function of capacitance for different gas mixtures. At the horizontal axis the real capacitance for one stage is plotted. The voltage of a stage is used as a parameter. As can be seen the highest energy input that can be obtained is not very dependent on the mixture. Only the $\mathrm{CO}_{2}: \mathrm{N}_{2}: \mathrm{He}=1: 1: 1$ mixture has a lower maximum for the energy input. When the maxi-

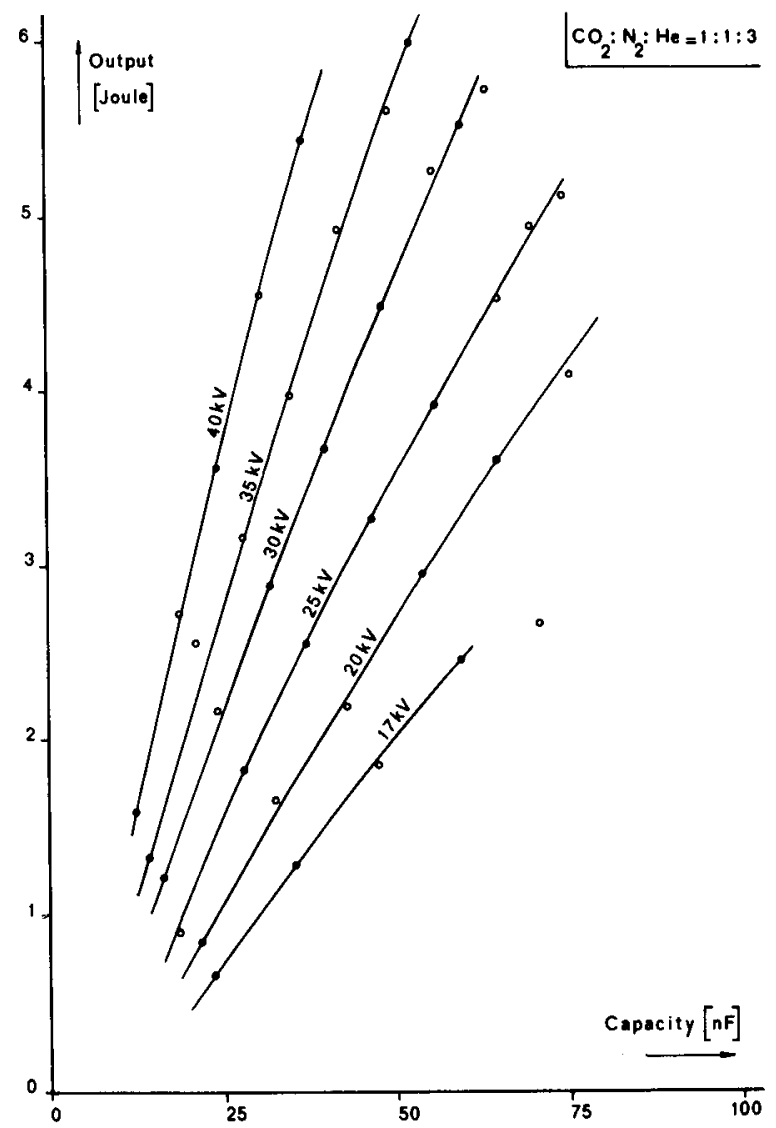

Fig. 5. The power output as a function of the capacitance of the Marx generator for a $\mathrm{CO}_{2}: \mathrm{N}_{2}: \mathrm{He}=1: 1: 3$ mixture. The voltage of the Marx generator is used as a parameter.

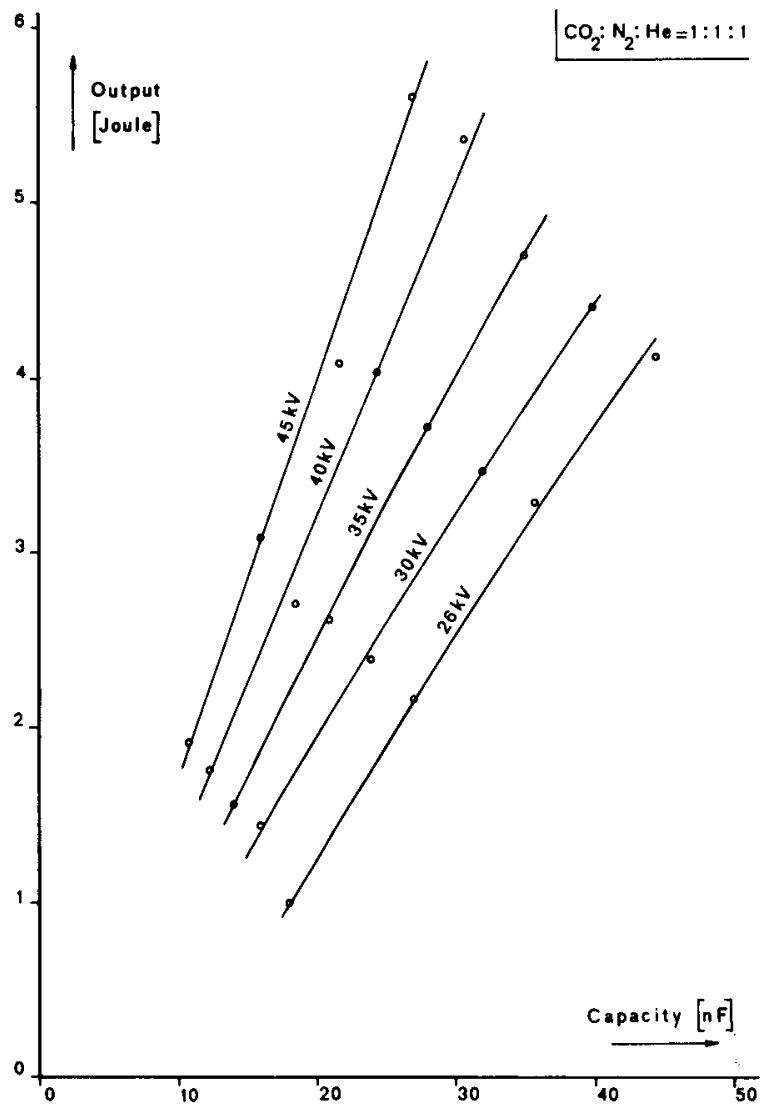

Fig. 6. The power output as a function of the capacitance of the Marx generator for a $\mathrm{CO}_{2}: \mathrm{N}_{2}: \mathrm{He}=1: 1: 1$ mixture. The voltage of the Marx generator is used as a parameter.

mum energy input is exceeded the laser usually does not spark between the electrodes, but numerous bright spots can be seen inside the active medium where the gas has a break down. The highest power output could be obtained with a $\mathrm{CO}_{2}: \mathrm{N}_{2}: \mathrm{He}=1: 2.5: 1.6$ mixture. We found a maximum energy output of $6.6 \mathrm{~J}$. For this case the output beam width was measured. A value of $17 \mathrm{~mm}$ was found. Thus the total active volume of $1.7 \times 2 \times 40 \mathrm{~cm}=135 \mathrm{~cm}^{3}$ produces a power output of $48 \mathrm{~J}$ per liter with an efficiency of $11.8 \%$. For lower voltages and especially for poor mixtures the efficiency can be somewhat better. The $\mathrm{CO}_{2}: \mathrm{N}_{2}: \mathrm{He}=$ 1:1:7 mixture reaches a $14.3 \%$ efficiency. Because the observed break down in the active medium appeared to be due to the optical break down (obviously the reflectivity of the outcoupling mirror was too high), we later on extended the measurements for the $1: 2.5: 1.6$ 


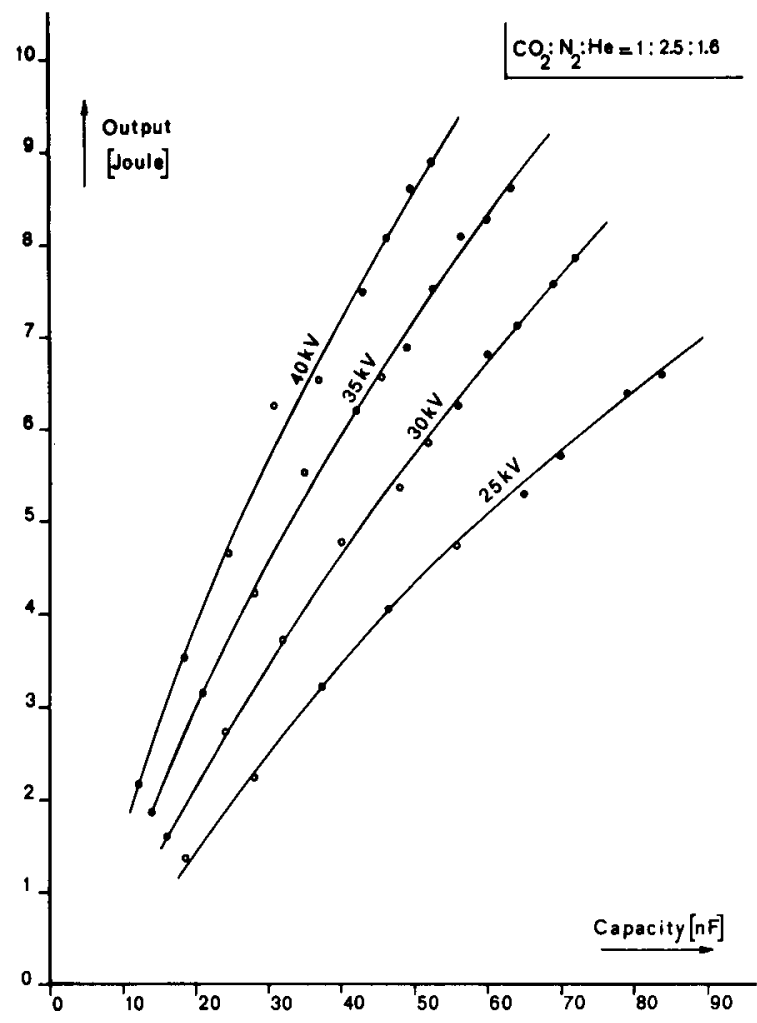

Fig. 7. The power output as a function of the capacitance of the Marx generator for a $\mathrm{CO}_{2}: \mathrm{N}_{2}: \mathrm{He}=1: 2.5: 1.6$ mixture. The voltage of the Marx generator is used as a parameter.

mixture. The closed circles in fig. 7 represent these extended measurements. Now a power output of $9 \mathrm{~J}$, i.e. more than $60 \mathrm{~J}$ per liter, could be reached with an efficiency of $10.6 \%$. Undoubtedly the measurements can also be extended to about the same power output level for the other mixtures. An example of the power output as a function of time can be seen in fig. 8 . The measurements have been performed with a photon drag detector. The time scale is $200 \mathrm{~ns} /$ div. A $1: 1: 3$ mixture is used in this case. The voltage applied and the capacitance per stage are $17 \mathrm{kV}$ and $60 \mathrm{nF}$ respectively. The rise time of the output is as short as 10 ns. The half-width is about $1 \mu \mathrm{s}$. For higher voltages we found the pulse duration to be somewhat shorter, obviously because of the lower impedance of the discharge or the lower capacitance of the Marx generator. The higher excitation rate may be the reason why a higher power output can be obtained at higher voltages.

We also performed some gain measurements. We

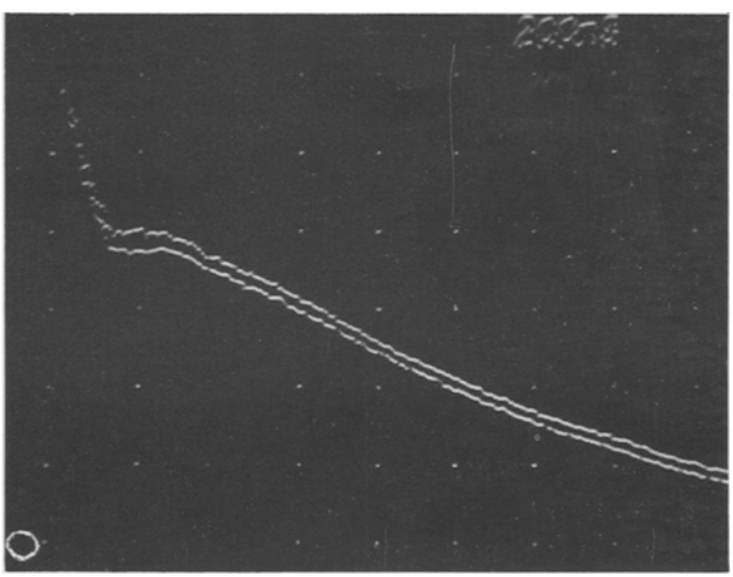

Fig. 8. The power output as a function of time. The time scale is $200 \mathrm{~ns} / \mathrm{div}$. The gas mix ture was $\mathrm{CO}_{2}: \mathrm{N}_{2}: \mathrm{He}=1: 1: 3$.

The Marx generator had a capacitance of $60 \mathrm{nF}$ and was loaded to $17 \mathrm{kV}$.

used the same construction as before, but the amplifier was now closed by two anti-reflection coated germanium windows. A stabilized $\mathrm{cw} \mathrm{CO}_{2}$ laser oscillating on the $\mathbf{P}(20)$ transition of the $001-100$ band and having a power output of a few Watts was used for probing. The continuous beam was chopped after the TEA am. plifier, so that we could continuously check its output level. A S.A.T.-type HgTe-CdTe diode detector having a rise time of $5 \mathrm{~ns}$ in connection with a transient digitizer from Tektronix was used for detecting the amplified signal. Fig. 9 shows a plot of the gain measure-

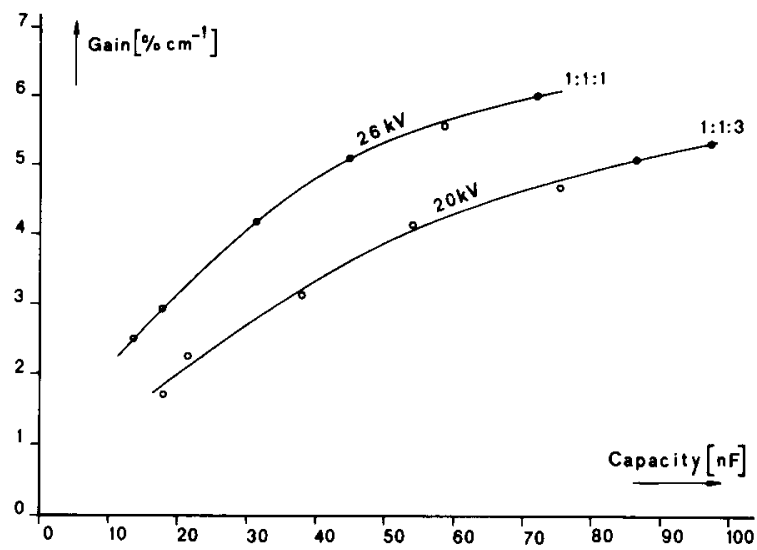

Fig. 9. The small-signal gain as a function of the capacity of the Marx generator. The upper curve is for a $1: 1: 1$ mixture with a Marx generator voltage of $26 \mathrm{kV}$. The lower curve is for a $1: 1: 3$ mixture with a voltage of $20 \mathrm{kV}$. 
ments for two different gas mixtures as a function of the real capacity value of one stage of the Marx generator. The upper curve represents the measurements for a $\mathrm{CO}_{2}: \mathrm{N}_{2}: \mathrm{He}=1: 1: 1$ mixture with a Marx-generator voltage of $26 \mathrm{kV}$ and the lower curve is for a $1: 1: 3$ mixture with a voltage of $20 \mathrm{kV}$. From the figure it can be seen that the gain saturates as a function of the capacitance. This probably has to do with the fact that the inversion production balances the spontaneous decay for large capacitance values. The largest gain value we measured was $6 \%$ per $\mathrm{cm}$ for a $1: 1: 1$ mixture. For this case the Marx generator was loaded to $26 \mathrm{kV}$ and had a capacitance value of $72 \mathrm{nF}$ per stage. A picture of this measurement is shown in fig. 10. The vertical axis gives the amplified signal of the cw probing beam. The unamplified signal has been set to one fifth of a division. This has been accomplished by setting the unamplified beam to one division exactly and then inserting a $20 \%$ transmitting Ge window in the beam just before the detector. So the amplification is 11 times over $40 \mathrm{~cm}$ length, which is in accordance with the

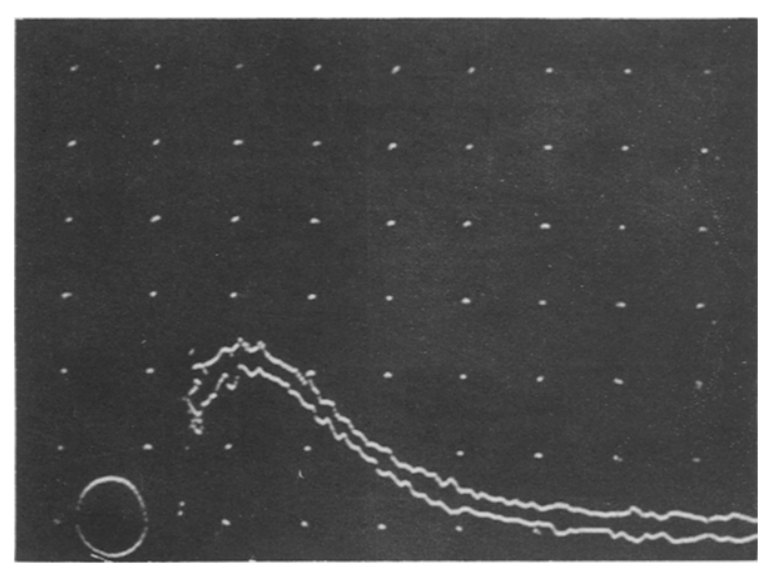

Fig. 10. The gain as a function of time. The time scale is 1 $\mu \mathrm{s} /$ div. The gas mixture was $\mathrm{CO}_{2}: \mathrm{N}_{2}: \mathrm{He}=1: 1: 1$. The capacitance of the Marx generator was $72 \mathrm{nF} /$ stage, loaded to $26 \mathrm{kV}$. For further explanation: see text. above-mentioned $6 \%$ per $\mathrm{cm}$. The time scale at the horizontal axis is $1 \mu$ s per division. Measurements of the gain of a pulsed $\mathrm{CO}_{2}$ laser having a pulse length of about 50 ns have given an exact confirmation of our measurements with the $\mathrm{cw}$ laser.

\section{Conclusions}

We have performed experiments on a new type of TEA $\mathrm{CO}_{2}$ laser. It differs from other systems by a very fast excitation rate. Since no separate UV source is present, the construction and alignment are very simple. The important feature that enables the high excitation rate is the built-in, very fast and strong UV source of large extend and excellent homogeneity. That is why a very homogeneous discharge is generated, resulting in a high energy output and a large small-signal gain. The quality of the output beam is excellent and the shot-to-shot reproducibility of the power output is within a few percent. An additional advantage of the fast rise time of the voltage pulse is the small time jitter that is present in the laser pulse, which can be used to advantage when the system is used in a chain of amplifiers.

The system can probably be applied with advan. tage to many other gas lasers. This has already been demonstrated for the case of $\mathrm{CO}$ [7].

\section{References}

[1] R. Dumanchin and J. Rocca-Serra, C.R. Acad. Sci. 269, sèrie $B(1969) 916$.

[2] A.J. Beaulieu, Appl. Phys. Letters 16 (1970) 504.

[3] G.J. Ernst, Ph. D. Thesis, Twente University of Technology, Enschede, The Netherlands, 1977.

[4] G.J. Ernst, Rev. Sci. Instr. 48 (1977) 1281.

[5] T.Y. Chang, Rev. Sci. Instr. 44 (1973) 405.

[6] A.J. Schwab and F.W. Hollinger, IEEE J. Quant. Electron QE-7 (1971) 484.

[7] P.J.M. Peters, Optics Commun. 25 (1978) 241. 